\title{
Evaluation of Different Signal Processing Methods in Time and Frequency Domain for Brain-Computer Interface Applications
}

\author{
J. Arnin, D. Kahani, H. Lakany and B. A. Conway
}

\begin{abstract}
Brain-computer interface (BCI) has been widely introduced in many medical applications. One of the main challenges in BCI is to run the signal processing algorithms in real-time which is challenging and usually comes with high processing unit costs. BCIs based on motor imagery task are introduced for severe neurological diseases especially locked-in patients. A common concept is to detect one's movement intention and use it to control external devices such as wheelchair or rehabilitation devices. In real-time BCI, running the signal processing algorithms might not always be possible due to the complexity of the algorithms. Moreover, the speed of the affordable computational units is not usually enough for those applications. This study evaluated a range of feature extraction methods which are commonly used for such realtime BCI applications. Electroencephalogram (EEG) and Electrooculogram (EOG) data available through IEEE Brain Initiative repository was used to investigate the performance of different feature extraction methods including template matching, statistical moments, selective bandpower, and fast Fourier transform (FFT) power spectrum. The support vector machine (SVM) was used for classification. The result indicates that there is not a significant difference when utilizing different feature extraction methods in terms of movement prediction although there is a vast difference in the computational time needed to extract these features. The results suggest that computational time could be considered as the primary parameter when choosing the feature extraction methods as there is no significant difference between the results when different features extraction methods are used.
\end{abstract}

\section{INTRODUCTION}

Brain-computer interface (BCI) is a technology with great potential as an assistive technology and to impact on a range of medical applications, including, diagnosis, treatment, and rehabilitation [1]. In patients with severe motor disabilities such as locked-in patients, BCI can play a role that enables those people to establish communication and environmental interaction without the necessity of functioning efferent pathways [2]. In rehabilitation, applications of BCI are also being used and developed to restore motor function by inducing activity-dependent brain plasticity and motor learning in addition to providing control over assistive devices that augment or restore movement and motor function lost after stroke or spinal cord injury [3].

In general, the standard BCI pipeline comprises of signal acquisition, signal preprocessing, digital signal processing and its application. Most of the BCI applications employ EEG and EOG which are non-invasive recording techniques.

J. Arnin, D. Kahani, H. Lakany and B. A. Conway are with the Centre of Excellence in Rehabilitation Engineering, Department of Biomedical Engineering, University of Strathclyde, 50 George St, Glasgow G1 1QE, United Kingdom (corresponding author, phone: +44 141548 3108; e-mail: jetsada.arnin@strath.ac.uk).
Many pieces of research reveal the advantages of EEG modalities such as a communication device based on steadystate visual evoked potential (SSVEP) [4] or mind-controlled wheelchair navigation using motor imagery (MI) [5]. Those applications can be operated offline or online (real-time) depending on the application. In real-time applications, there are significant concerns when implementing a BCI and include accuracy of prediction, latency, consistency, and flexibility. These issues also relate to design limitations associated with hardware specification, the complexity of an algorithm, and multi-domain signal processing requirements (time and frequency). The most critical consideration in BCI processing is computation time, and general computing hardware is not fast enough for real-time applications [6]. With current technology, there is a trend towards using hardware acceleration units in scientific computing applications requiring real-time solutions [7-8] and this approach can increase the feasibility of using sophisticated algorithms or multi-domain processing in high-speed BCIs.

Regarding classification, training the classifier is computationally expensive, but it does not need to be run in real-time since an offline process can complete it. Consequently, the most crucial part is the real-time feature extraction. In this study, we evaluated the difference between a range of time-domain and frequency-domain feature extraction approaches for further execution using hardware acceleration. The study used an open-access dataset for comparing the performance of proposed feature extraction methods in terms of accuracy and computation time.

\section{ARCHIVE EEG DATASET}

\section{A. Selection Criteria}

An archive EEG dataset was employed to investigate the performance of different feature extraction approaches. As many open-source EEG datasets are available via open access for BCI research, we applied the following selection criteria in choosing datasets for this study.

Data Quality: The datasets must have sufficient sampling rate (generally more than $250 \mathrm{~Hz}$ ). It should have good signal-to-noise ratio and follow a clear protocol.

Usability: The datasets should have adequate numbers of recruited participants, and the selected data should have public engagement with high impact and citation.

Familiarity: The datasets should relate to our laboratory's field of knowledge and experimental expertise, primarily human neurophysiology and motor control.

Compatibility: The data format of the selected dataset should be general and usable on multiple platforms. In addition, the EEG channel montage used should be appropriate for motor imagery recordings. 
Using these criteria, we selected the dataset IV from Brain/Neural Computer Interaction: Horizon 2020 [9]. This dataset related to motor imagery tasks commonly used in our laboratory.

According to the dataset [9], the subjects were asked to randomly perform 2-class motor imagery (left and right) with a directional visual cue presented on a screen. The EEG and EOG data were collected from 9 healthy participants. There were five sessions each with 120 repetitions. The sampling frequency was $250 \mathrm{~Hz}$ with a dynamic range of \pm 250 microvolts. Analog bandpass filtering $(0.5-100 \mathrm{~Hz})$ and a notch filter at $50 \mathrm{~Hz}$ were used. The recording electrodes were placed at $\mathrm{C} 3, \mathrm{C} 4$, and $\mathrm{Cz}$ according to the international 10-20 system. The original authors processed the data and demonstrated results based on a general signal processing pipeline including signal pre-processing, feature extraction and selection, binary classification and error calculation. Fig. 1 shows the overall protocol of motor imagery task used to record this dataset.

Figure 1. (a) Diagram of cue-based motor imagery task from the selected dataset [9]. Each single-trial lasted 7 seconds which the cue appeared at 3 seconds after starting. The figure is adapted from the original publication.

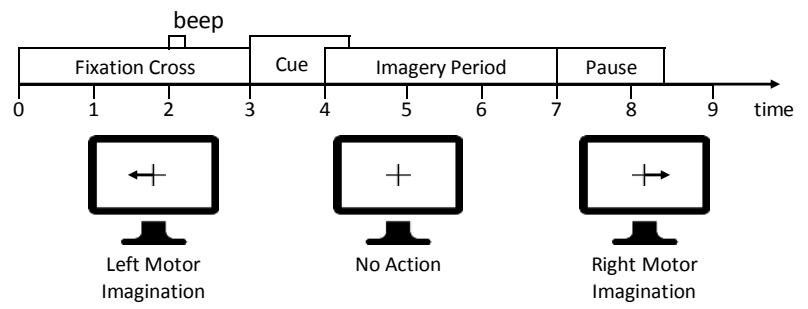

\section{B. Data Visualization}

First, we explored the overall data in both time and frequency domains using the continuous wavelet transform (CWT) based on Morlet wavelet [10] as shown in Fig. 2. visualization was performed across all data recorded from all subjects. The results demonstrate increased $8-15 \mathrm{~Hz}$ activity occurring around the time of the movement cue.

Figure 2. Data visualization in time-frequency analysis of $\mathrm{C} 3$ and $\mathrm{C} 4$ position taken from an anonymous subject.
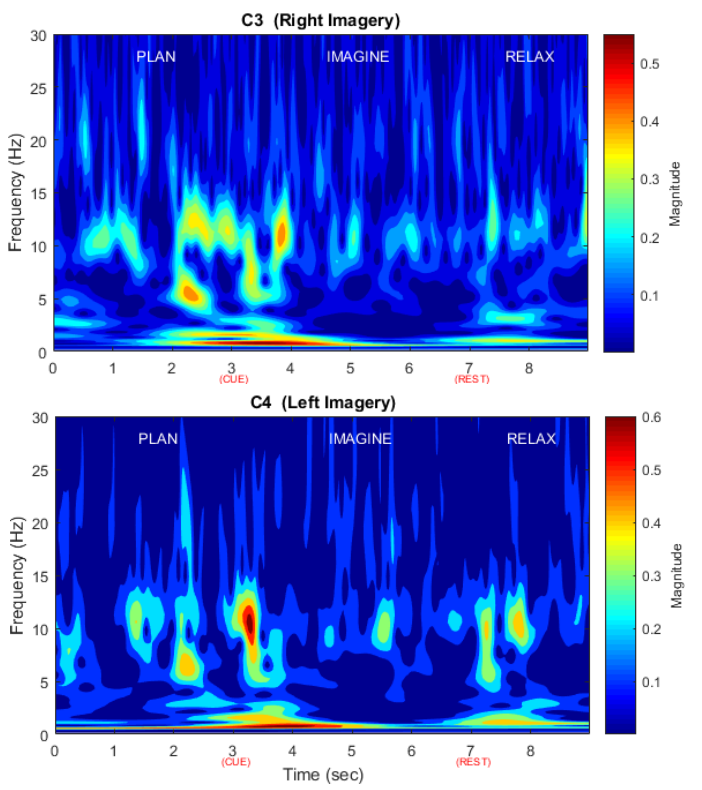

\section{Proposed Signal Processing Chain}

Fig. 3 illustrates the overall block diagram of our signal processing pipeline. After exploring the dataset, artifacts were reduced using independent component analysis (ICA) which will be described in session III. The 'artifact-corrected' EEG was processed using different feature extraction methods. Thereafter, the classification error was calculated to demonstrate the performance of those features.

Figure 3. Proposed signal processing chain.

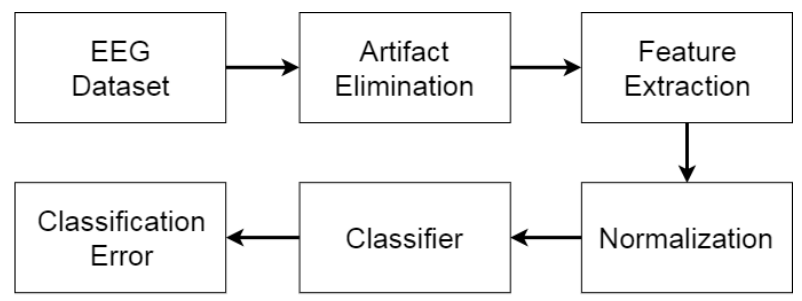

\section{ICA-BASED ARTIFACT ELIMINATION}

R. Leeb and et al. [9] used regression analysis for artifact removal in the dataset. In regression analysis, the assumption is that the recorded EEG has a linear relationship between the real EEG signal and the spatial EOG components. In this work, we used independent component analysis (ICA) technique to reduce contamination by unwanted signals as this yields results better than regression method [11]. Since the dataset provides three channels of both EEG and EOG, those signal were used in ICA decomposition. In this study, the EOG recorded at the frontal site was used in ICA to remove the artifacts from the recorded EEG channels. The result of using ICA technique is shown in Fig. 4 and produced an effective removal of the EOG artifact. It should be noted that in the original published data the results of artifact reduction have been observed by an expert and trials having artifacts were marked to report the results.

Figure 4. Artifact-free EEG using ICA decomposition.
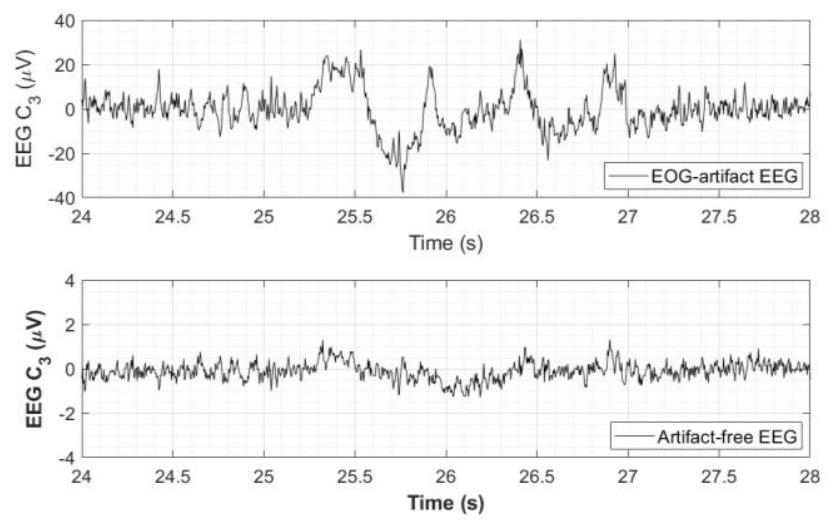

\section{FEATURE EXTRACTION}

To examine the classification success rate using different feature extraction methods, we used four common feature extraction methods all of which are widely used in BCI applications including template matching [12-13], statistical moments [14-15], selective bandpower [16-17] and fast Fourier transform (FFT) power spectrum [18-19]. It should be noted that the original work used the selective bandpower as a feature vector. 


\section{A. Template Matching}

Template matching is a time-domain computation method which measures the similarity between incoming data and an existing template using the dot product of two data. This can be explained using cross-correlation function by

$$
r_{x y}[\tau]=\frac{1}{N} \sum_{n=0}^{N-1} x[n] \cdot y[n-\tau]^{*} .
$$

Where $x[n]$ is an incoming signal and $y[n]$ is a template. In this study, we calculated the template by epoching the EEG data around the cue point and averaging them for each motor imagery task. The averaged data was used as a template to measure the correlation. The similarity measurement was then used as a vector feature to train an SVM classifier.

\section{B. Statistical Moments}

Statistical moments are specific quantitative measurements in time domain analysis. The common form of the n-th order statistical moment can be described as

$$
\frac{\mu_{n}}{\sigma^{n}}=\frac{E\left[(x-\mu)^{n}\right]}{\sigma^{n}} .
$$

In this study, the feature vector composed is a vector of two entries extracted from only the first order and second order moments. Adding higher order moments to the feature vector can improve the classification result, but it comes at the expense of having more training samples. Hence, it was not possible to use the dataset used in this study for the higher order.

\section{Selective Bandpower}

The time-domain average bandpower can be estimated by filtering the incoming EEG, squaring and averaging the samples over the past second as proposed in [9]. The feature vector was composed of this outcome. The general form of bandpower calculation can be defined as

$$
B P_{\text {selective }}=\frac{1}{N} \sum_{n=0}^{N-1}(x[n] * h[n])^{2} .
$$

The $\mathrm{h}[\mathrm{n}]$ is an impulse response of the bandpass filter for convolution with $x[n]$ which in this study was fixed at 7-22 $\mathrm{Hz}$ according to the pervious study [9].

\section{FFT Power Spectrum}

The FFT frequency analysis is a well-established technique commonly used for EEG frequency EEG analysis. The general Fourier transformation equation is

$$
X\left[e^{j \omega}\right]=\frac{1}{N} \sum_{n=0}^{N-1} x[n] e^{-j \omega n},
$$

The feature vector element was taken from the relative power spectrum of the signal which can be described as

$$
P_{\text {relative }}=\frac{\int_{f_{1}}^{f_{2}} X\left[e^{j 2 \pi f}\right] d f}{\int_{-\infty}^{\infty} X\left[e^{j 2 \pi f}\right] d f} .
$$

The bandwidth frequency $\mathrm{f}_{1}$ and $\mathrm{f}_{2}$ were also fixed to 7 and $22 \mathrm{~Hz}$, respectively to compare the outcome with the time-domain selective bandpower method.

\section{Classification}

Linear discriminant analysis (LDA) was used by R. Leeb and et al. [9] for dimensionality reduction before binomial classification that yielded the average classification error of
$25 \%$ for the offline analysis. As an alternate approach and to utilize of good generalization properties, and insensitivity to overtraining [20], this study used SVM to categorize the data. In relation to SVM, various kernel functions including a linear kernel, quadratic kernel, polynomial kernel and Gaussian kernel were performed to select the best result. The proposed features were initiated as a feature vector. In this study, we used the data only from C3 and C4 to create a twodimensional feature vector. This means that the single vector is the elements of the obtained outcome of the training set which were concatenated from all trials. The feature vector was processed in SVM by using data windowing over 0-4 second following cue occurrence. The window length was set to 1,000 milliseconds, and the window was moved sample by sample in order to calculate classification errors. The classification was performed with $10 \times 10$ cross-validation technique, and the average maximum accuracy was described as well as its time point for comparison with the original work. In addition, the computation time of each feature operated on through MATLAB programming using a singlecore CPU (without hardware acceleration unit) was reported.

\section{RESUlt AND DISCUSSION}

After data interpretation, four different types of feature extraction methods were used to extract features from the provided dataset. The SVM kernel was selected by trial and error. Also, the $10 \times 10$ cross-validation was performed to verify the consistency of SVM classification, and then the average maximum accuracy was obtained. The results are reported regarding classification error and computation time in Table I and Fig. 5. There was no significant difference observed using different features extraction methods ( $p$ value $>0.05$ ). The analysis of variance revealed there was no best feature extraction method based on the classification prediction rate. It should be noted that feature extraction for every individual subject has to be fine-tuned for better results but for consistency, we considered the parameters the same for all the subjects. According to the original work [9], the average classification error is $25.0 \pm 11.7 \%$. For the computation time, this study used MATLAB running on 64 bit Window 10 operating system, with 16 GB of DDR3

\begin{tabular}{|c|c|c|c|c|}
\hline \multirow{2}{*}{ Subject } & \multicolumn{4}{|c|}{ Classification Error ${ }^{a}(\%)$} \\
\hline & Template & S-Moment & $S u b-B P$ & FFT Power \\
\hline 1 & 27.2 & 36.8 & 31.2 & 32.4 \\
\hline 2 & 32.4 & 34.8 & 39.9 & 35.4 \\
\hline 3 & 35.2 & 29.3 & 39.2 & 43.3 \\
\hline 4 & 39.5 & 36.3 & 6.7 & 9.8 \\
\hline 5 & 20.6 & 28.1 & 37.1 & 21.7 \\
\hline 6 & 37.4 & 30.0 & 24.9 & 26.2 \\
\hline 7 & 23.5 & 26.2 & 25.5 & 21.9 \\
\hline 8 & 38.0 & 36.0 & 22.0 & 22.4 \\
\hline 9 & 39.9 & 40.6 & 26.2 & 31.0 \\
\hline Mean & $32.6 \pm 7.2$ & $33.1 \pm 4.8$ & $28.1 \pm 10.4$ & $27.1 \pm 9.7$ \\
\hline
\end{tabular}
memory and Intel Core i7-6700 processors at 2.6 GHz. Note that the algorithms were executed on single-core processing unit without acceleration and optimization.

TABLE I. ClassificAtion ERror of Four DifFERENT FEATURES 
Regarding the decision period of subject's imagination, the results were reported in Table II. The average event duration is about $2.52 \pm 0.51$ seconds after the cue. This outcome is close to the results reported by original publication [9] estimate of $2.88 \pm 0.51$ seconds.

TABLE II. DECISION DURATION OF Four DifFERENT FeAtures

\begin{tabular}{|c|c|c|c|c|}
\hline \multirow{2}{*}{ Subject } & \multicolumn{4}{|c|}{ Decision Duration $^{\text {a }}$ (s) } \\
\cline { 2 - 5 } & Template & S-Moment & Sub-BP & FFT Power \\
\hline 1 & 2.38 & 1.48 & 2.58 & 2.40 \\
\hline 2 & 1.73 & 1.74 & 2.63 & 2.33 \\
\hline 3 & 2.50 & 2.97 & 2.46 & 2.62 \\
\hline 4 & 1.92 & 3.26 & 3.12 & 2.73 \\
\hline 5 & 2.62 & 2.82 & 3.82 & 2.37 \\
\hline 6 & 2.13 & 1.72 & 2.70 & 2.87 \\
\hline 7 & 2.59 & 2.83 & 2.47 & 2.36 \\
\hline 8 & 2.08 & 1.50 & 2.98 & 2.70 \\
\hline 9 & 2.52 & 3.10 & 2.75 & 3.13 \\
\hline Mean & $2.28 \pm 0.32$ & $2.38 \pm 0.75$ & $2.83 \pm 0.43$ & $2.61 \pm 0.28$ \\
\hline \multicolumn{5}{|c}{ a. There is no significant difference between groups (p-value >0.05). }
\end{tabular}

Figure 5. Results of compared computation time (in microsecond) of each feature operating on MATLAB programming using single-core processing.

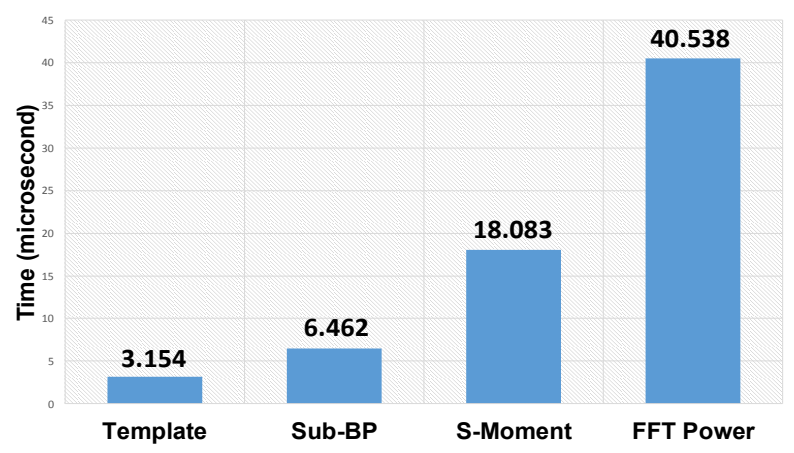

\section{CONCLUSION}

In conclusion, we demonstrated the difference between time and frequency domain approaches for a two-directional imaginary motor movement task. Our significant findings reveal that using the different feature extraction methods did not result in a significant difference in the classification accuracy but only in the computation time. For example, FFT methods consume much more time than others without offering accuracy advantage. The results highlight that computation time should be considered as a primary outcome when selecting algorithms for use in real-time applications and also highlight the utility and advantages of using open-access data archives for comparative BCI research.

\section{ACKNOWLEDGMENT}

The authors would like to acknowledge funding from Scottish Government Health Directorates and the Royal Thai Government scholarship.

\section{REFERENCES}

[1] A. Vallabhaneni, T. Wang, and B. He, "Brain-computer interface," in Neural engineering: Springer, 2005, pp. 85-121.

[2] R. Rupp, "Challenges in clinical applications of brain computer interfaces in individuals with spinal cord injury," Frontiers in neuroengineering, vol. 7, p. 38, 2014.

[3] E. Buch et al., "Think to move: a neuromagnetic brain-computer interface (BCI) system for chronic stroke," Stroke, vol. 39, no. 3, pp. 910-917, 2008.

[4] P. Martinez, H. Bakardjian, and A. Cichocki, "Fully online multicommand brain-computer interface with visual neurofeedback using SSVEP paradigm," Computational intelligence and neuroscience, vol. 2007, 2007.

[5] D. Huang et al., "Electroencephalography (EEG)-based braincomputer interface (BCI): A 2-D virtual wheelchair control based on event-related desynchronization/synchronization and state control," IEEE Transactions on Neural Systems and Rehabilitation Engineering, vol. 20, no. 3, pp. 379-388, 2012.

[6] S. Aluru and N. Jammula, "A review of hardware acceleration for computational genomics," IEEE Design \& Test, vol. 31, no. 1, pp. 19$30,2014$.

[7] N. Wang et al., "Accelerated Rendering and Fast Reconstruction of EEG Data in Real-Time BCI," in Proceedings of the 2015 Chinese Intelligent Automation Conference, 2015, pp. 61-75: Springer.

[8] P. Dohnálek et al., "Pattern recognition in EEG cognitive signals accelerated by GPU," in International Joint Conference CISIS'12ICEUTE' 12-SOCO' 12 Special Sessions, 2013, pp. 477-485: Springer.

[9] R. Leeb et al., "Brain-computer communication: motivation, aim, and impact of exploring a virtual apartment," IEEE Transactions on Neural Systems and Rehabilitation Engineering, vol. 15, no. 4, pp. 473-482, 2007.

[10] H. Adeli, Z. Zhou, and N. Dadmehr, "Analysis of EEG records in an epileptic patient using wavelet transform," Journal of neuroscience methods, vol. 123, no. 1, pp. 69-87, 2003.

[11] T.-P. Jung et al., "Removing electroencephalographic artifacts by blind source separation," Psychophysiology, vol. 37, no. 2, pp. 163$178,2000$.

[12] A. Aarabi et al., "Detection of EEG transients in neonates and older children using a system based on dynamic time-warping template matching and spatial dipole clustering," Neuroimage, vol. 48, no. 1, pp. 50-62, 2009.

[13] H. Qu and J. Gotman, "A patient-specific algorithm for the detection of seizure onset in long-term EEG monitoring: possible use as a warning device," IEEE transactions on biomedical engineering, vol. 44, no. 2, pp. 115-122, 1997.

[14] S. S. Soliman and S.-Z. Hsue, "Signal classification using statistical moments," IEEE Transactions on Communications, vol. 40, no. 5, pp. 908-916, 1992.

[15] S. S. Alam and M. I. H. Bhuiyan, "Detection of seizure and epilepsy using higher order statistics in the EMD domain," IEEE journal of biomedical and health informatics, vol. 17, no. 2, pp. 312-318, 2013.

[16] G. Pfurtscheller et al., "Mu rhythm (de) synchronization and EEG single-trial classification of different motor imagery tasks," Neuroimage, vol. 31, no. 1, pp. 153-159, 2006.

[17] R. Palaniappan, "Brain computer interface design using band powers extracted during mental tasks," in Neural Engineering, 2005. Conference Proceedings. 2nd International IEEE EMBS Conference on, 2005, pp. 321-324: IEEE.

[18] K.-E. Ko, H.-C. Yang, and K.-B. Sim, "Emotion recognition using EEG signals with relative power values and Bayesian network," International Journal of Control, Automation and Systems, vol. 7, no. 5, p. $865,2009$.

[19] C. Lehmann et al., "Application and comparison of classification algorithms for recognition of Alzheimer's disease in electrical brain activity (EEG)," Journal of neuroscience methods, vol. 161, no. 2, pp. 342-350, 2007.

[20] A. Subasi and M. I. Gursoy, "EEG signal classification using PCA, ICA, LDA and support vector machines," Expert Systems with Applications, vol. 37, no. 12, pp. 8659-8666, 2010. 